\title{
ACTIVIDAD ESTROGÉNICA DEL EXTRACTO HIDROALCOHÓLICO DEL FRUTO DE AGUAJE MAURITIA FLEXUOSA L EN RATAS OVARIECTOMIZADAS
}

\author{
Karen Elizabeth Campos Correa ${ }^{1}$, Cynthia Joyce Gutiérrez Landa ${ }^{1}$
}

\begin{abstract}
RESUMEN
Objetivo. Determinar la actividad estrogénica del extracto hidroalcohólico del fruto de aguaje (Mauritia flexuosa L) en ratas ovariectomizadas. Materiales y métodos. 54 ratas hembras Sprague-Dawley de $2 \frac{1}{2}$ a 3 meses de edad fueron divididas aleatoriamente en seis grupos: hembras intactas + suero, ovariectomizadas + suero, ovariectomizadas + extracto hidroalcohólico de aguaje a tres dosis diferentes, ovariectomizadas + etinilestradiol. Después de la ovariectomía se inició el tratamiento por vía intragástrica durante 5 semanas. A las 2 semanas de tratamiento se hizo un frotis vaginal por 7 días para determinar la maduración celular. Al finalizar el tratamiento se realizó la eutanasia para estudiar el tejido óseo de la metáfisis proximal de la tibia; y se disecó el útero para su pesado. Resultados. El grupo que recibió la dosis de $500 \mathrm{mg} / \mathrm{kg}$ presentó un mayor número de ratas que tuvieron maduración celular vaginal a diferencia de las dosis menores. En el estudio del hueso no hubo actividad estrogénica significativa en el hueso trabecular pero se obtuvo un aumento significativo de osteoblastos en los grupos tratados con el extracto hidroalcohólico de aguaje a dosis de $100 \mathrm{mg} / \mathrm{kg}$. El peso uterino de los grupos tratados con el extracto y las ovariectomizadas con suero fueron similares. Conclusiones. El extracto hidroalcohólico del fruto de aguaje presentó actividad estrogénica en ratas ovariectomizadas en las células vaginales; la dosis de $100 \mathrm{mg} / \mathrm{kg}$ produjo un aumento significativo del número de osteoblastos, y no se evidenció actividad estrogénica en el peso uterino con ninguna de las dosis del extracto hidroalcohólico de aguaje.
\end{abstract}

Palabras clave: Aguaje; Mauritia Flexuosa L; Actividad estrogénica; Ratas ovariectomizadas (fuente: DeCS BIREME).

\section{ESTROGENIC ACTIVITY OF THE HYDROALCOHOLIC EXTRACT OF THE FRUIT OF AGUAJE MAURITIA FLEXUOSA L IN OVARIECTOMIZED RATS}

\begin{abstract}
Objectives. To determine the estrogenic activity of hydroalcoholic extract of the fruit of aguaje (Mauritia flexuosa L) in ovariectomized rats. Materials and methods. 54 Sprague-Dawley rats from $21 / 2$ to 3 months of age were grouped randomly into six groups: intact females + saline, ovariectomized + saline, hydroalcoholic extract of three different doses aguaje, ovariectomized + ethinylestradiol. After ovariectomy treatment was started by intragastric for 5 weeks. At 2 weeks of treatment a vaginal smear was made for 7 days to determine the cell maturation. At the end of the treatment was performed euthanasia to study the bone tissue of the proximal metaphysis of the tibia and the uterus was dissected for weighing. Results. The group that received doses of $500 \mathrm{mg} / \mathrm{kg}$ had more rats that had vaginal cell maturation in contrast to the lower dose. In the study of bone was not significant estrogenic activity in trabecular bone but it was a significant increase in osteoblasts in the groups treated with the hydroalcoholic extract of aguaje at doses of $100 \mathrm{mg} / \mathrm{kg}$. Uterine weight in the groups treated with the extract and saline were similar ovariectomized. Conclusions. The hydroalcoholic extract of the fruit of aguaje presented in ovariectomized rats estrogen activity in cells vaginal dose of $100 \mathrm{mg} / \mathrm{kg}$ produced a significant increase in the number of osteoblasts, and no evidence of estrogenic activity in uterine weight with any of the hydroalcoholic extract dose aguaje.
\end{abstract}

Key words: Aguaje; Mauritia flexuosa L; Estrogenic activity; Ovariectomized rats (source: MeSH NLM).

\section{INTRODUCCIÓN}

El aguaje Mauritia flexuosa $\mathrm{L}$, es una palmera con amplia distribución en el Perú y otros países de América del Sur, creciendo principalmente en territorios de clima tropical. La principal virtud de esta palmera son sus frutos ( de los cuales se usa el mesocarpio o pulpa) que sirven como alimento en muchas zonas amazónicas, ya sea rurales, urbanas o en tribus de indígenas ${ }^{1}$. Con respecto al fruto hay creencias populares que le atribuyen virtudes hormonales por influir en los caracteres sexuales femeninos, y disminuir las molestias propias del climaterio. Es probable que este fruto contenga fitoestrógenos, que son compuestos con similar estructura a los estrógenos, pero con efecto menor ${ }^{2}$. Uno de los usos de fitoestrógenos sería en la etapa de la postmenopausia, ya que no se ha reportado, los efectos adversos que puede producir la Terapia de Reemplazo Hormonal (TRH), como los accidentes cerebrovasculares, enfermedades coronarias, infartos al corazón y desarrollo de cáncer de mama y endometrio ${ }^{3-4-5}$. Si bien existen estudios acerca de los compuestos antes mencionados (especialmente sobre los fitoestrógenos de la soya), las investigaciones son escasas con respecto al aguaje y sus propiedades

Licenciada en Obstetricia. Egresada de la Universidad Nacional Mayor de San Marcos. Ex-Interna del Instituto Nacional Materno Perinatal. Lima - Perú

Recibido: 10-10-12 Aprobado: 22-04-13 
estrogénicas; si dichas propiedades son comprobadas ofreceríamos bases científicas para estudios posteriores (sobre su toxicología, farmacocinética, farmacodinamia, eficacia y mecanismos de acción) cuyos resultados podrían extender el consumo de este fruto hacia otras poblaciones así como su comercialización, mejorando la salud de las mujeres y poniendo al alcance de ellas las propiedades que este producto ofrece. El objetivo de la presente investigación es demostrar la actividad estrogénica del extracto hidroalcohólico del fruto de aguaje en ratas ovariectomizadas, utilizando como indicadores a la citología vaginal, tejido óseo y peso uterino.

\section{MATERIALES Y MÉTODOS}

\section{Diseño de Investigación}

Tipo de investigación: Experimental-Preclínico Unidad de análisis: Ratas hembras Sprague-Dawley de 2 $1 / 2$ a 3 meses.

Tamaño de la muestra: 54 ratas hembras Sprague-Dawley de $21 / 2$ a 3 meses.

\section{Asignación de Grupo: Aleatorio Simple}

Variable Independiente: Extracto hidroalcohólico del fruto de aguaje

\section{Variable Dependiente: Efecto estrogénico.}

Criterios de inclusión: Ratas Sprague-Dawley de 2 1/2 a 3 meses de edad, hembras y en buen estado general.

Criterios de exclusión: Ratas menores de 2 1/2 de edad o mayores de 3 meses de edad, hembras preñadas, en mal estado general y las que murieran durante el estudio.

\section{Procedimiento:}

a) Preparación del extracto hidroalcohólico del fruto de aguaje (EHA): Se adquirió el aguaje, morfotipo "amarillo" en el mercado Modelo Mayorista de Frutas (Lima) procedente principalmente del Departamento de San Martín. Los frutos fueron lavados con agua potable y luego remojados en agua caliente por 4 horas en recipientes de acero con la finalidad de acelerar el proceso de maduración, hasta que el pericarpio pudiera retirarse con la yema de los dedos sin mayor esfuerzo. Se separó la pulpa para la preparación del extracto. Cada $200 \mathrm{~g}$ de pulpa fueron licuados con $450 \mathrm{ml}$ de alcohol al 70\%. Esta mezcla se depositó en un envase ámbar herméticamente cerrado por una semana para su maceración; posteriormente se procedió a su filtración, para lo cual se utilizó una bomba de filtración al vacío. El filtrado se depositó en un recipiente de vidrio que fue puesto en una estufa a $40^{\circ} \mathrm{C}$ por dos semanas para la evaporación de la parte líquida y así obtener el extracto seco. Para la preparación de las 3 concentraciones de EHA se pesó el extracto seco y se diluyó con agua destilada.

b) Preparación y agrupación de los animales: Las ratas Sprague Dawley fueron adquiridas del Instituto Nacional de Salud (INS) y alojadas en jaulas de crianza del Bioterio de la Facultad de Medicina de la Universidad Nacional Mayor de San Marcos. Permanecieron en un ambiente de 20 a $22{ }^{\circ} \mathrm{C}$ de temperatura, 50 a $60 \%$ de humedad y $12 \mathrm{~h}$ de luz/ oscuridad, teniendo además acceso libre del agua y alimento balanceado. Luego de una semana de adaptación, se asignaron aleatoriamente los 6 grupos de estudio: Grupo $1(\mathrm{n}=9)$ : ratas hembras intactas + suero fisiológico $1 \mathrm{ml} / 100 \mathrm{gr}$; Grupo $2(\mathrm{n}=9)$ : OVX + suero fisiológico $1 \mathrm{ml} / 100 \mathrm{gr}$; Grupo $3(\mathrm{n}=9)$ : OVX + EHA de 10 mg/kg; Grupo 4 (n=9): OVX + EHA $100 \mathrm{mg} /$ kg; Grupo 5 (n=9): OVX + EHA 500 mg/kg; Grupo 6 $(\mathrm{n}=9):$ OVX + Etinilestradiol $0,1 \mathrm{mg} / \mathrm{kg}$.

c) Ovariectomía de las ratas: Con la ovariectomía (intervención quirúrgica para extraer los ovarios), se puede evaluar la influencia del estrógeno a utilizar. Para dicha operación se utilizaron materiales esterilizados y se anestesió a las ratas con ketamina de $50 \mathrm{mg} / \mathrm{kg}$ vía intraperitoneal. Previo rasurado del área lumbar lateral se hizo una incisión horizontal de $1 \mathrm{~cm}$ aproximadamente para ubicar el ovario de dicha zona, se ligó con catgut crómico para extirparlo y evitar hemorragias. Se suturó la incisión empleando seda negra 1/0; este procedimiento fue repetido en la zona contralateral.

d) Preparación de láminas: Para la lectura del frotis vaginal se utilizó la coloración de Papanicolaou; y para el estudio del tejido óseo se realizó un corte longitudinal a la altura de la metáfisis proximal de la tibia de 3-4 $\mu \mathrm{m}$ de grosor con ayuda de un micrótomo para su lectura se empleó la coloración de Hematoxilina Eosina.

\section{e) Valoración de resultados}

Frotis del epitelio vaginal: Se realizó un frotis vaginal diariamente entre 9:00 - 11:00 am por 7 días después de 2 semanas de tratamiento, con la finalidad de evaluar la madurez de células vaginales a través del índice de maduración celular donde se identifican a las células parabasales (células inmaduras), intermedias y superficiales. Ya que el ciclo estral de la rata dura entre 4 a 5 días, se asignó un tiempo de 7 días para el frotis vaginal y así aumentar la probabilidad de encontrar células maduras de cada etapa estral si es que lo hubiera.

Luego de 10 a 15 días post-ovariectomía los frotices de las ratas se encuentran en fase inactiva, es decir solo se observa células inmaduras ${ }^{6}$.

Si un frotis tiene de $70 \%$ a más de células parabasales se interpreta como ausencia de estrógenos ${ }^{7}$; y 
si el porcentaje de parabasales es menor a $70 \%$ corresponde a la presencia de actividad estrogénica. En cada fase del ciclo estral el porcentaje de los tipos celulares son distintos pero en las fases de mayor actividad estrogénica se evidencian las células intermedias y superficiales en mayor proporción.

Un total de 378 láminas fueron leídas por un médico patólogo, la cual desconocía al grupo que pertenecían las muestras; utilizó un microscopio electrónico LEICA DM 500.

Estudio del Tejido Óseo: La zona de estudio fue el hueso trabecular de la metáfisis proximal de la tibia derecha de cada animal, ya que luego de 5 semanas de la ovariectomía, en un modelo de osteoporosis en ratas, se ha encontrado resultados estadísticamente significativos en dicha zona tras la depleción estrogénica ${ }^{8-9}$

Para la evaluación del tejido óseo se hizo un estudio histomorfométrico analizando el hueso trabecular $y$ los osteoblastos. En el primero se evaluó: número de trabéculas en un campo de $10 \mathrm{X}$, proporción trabecular medido en porcentaje (\%) y grosor trabecular medido en micras $(\mu \mathrm{m})$; y para los osteoblastos se realizó un conteo de estas células en las trabéculas en un campo de $40 \mathrm{X}$. Para la lectura de las láminas se utilizó un microscopio electrónico Modelo OLYMPUS CX 41 ello estuvo a cargo de un médico patólogo el cual no sabía a qué grupo pertenecían las muestras; también se utilizó un programa de medición computarizada J Micro Vision versión 1,2,7.

Determinación de peso uterino: Posteriormente a la eutanasia se extrajo el útero de las ratas, para disecarlas e inmediatamente pesarlas en una balanza de alta precisión Modelo SARTORIUS BP121S. Este parámetro evalúa indirectamente la atrofia o hipertrofia del útero en los animales de estudio.

Análisis Estadístico: Para el análisis de los datos se empleó el Paquete Estadístico SPSS Versión 18. En el análisis del frotis vaginal se utilizaron estadísticas descriptiva, prueba de normalidad de KolmogorovSmirnov; el ANOVA para comparaciones múltiples y Post Hoc DMS; además se usó la Prueba de Chi cuadrado. En el análisis del tejido óseo (hueso trabecular y osteoblastos) se emplearon estadísticas descriptivas, la prueba de normalidad de Kolmogorov-Smirnov; y para las comparaciones múltiples, a los que tuvieron una distribución normal se les aplicó la prueba ANOVA y Post Hoc DMS; y los parámetros que no tuvieron una distribución normal se les realizó la prueba de KruskalWallis y Post Hoc Dunn, con un $95 \%$ de confianza, $\alpha=0,05$. Para el peso uterino se aplicó estadísticas descriptivas.

Aspectos éticos: Tuvieron una semana de adaptación para reducir el estrés del animal; se controló los factores ambientales como temperaturas extremas, concentraciones altas de amoníaco por la orina; a través de un ambiente ventilado, sin ruido y limpio. Además se utilizó el mínimo animal necesario por jaula, en beneficio de la investigación. Para el sacrificio del animal elegimos el método de "Eutanasia inducido por anestésicos inhalatorios"; este es un método aceptable para roedores ${ }^{10}$. El método consiste en embeber una torunda de algodón con éter etílico, para colocarlo en el interior de una campana de vidrio junto al animal y esperar su muerte; esto permite una rápida pérdida de conciencia sin dolor, seguido por un alivio progresivo hasta la muerte del animal.

\section{RESULTADOS}

\section{Frotis vaginal}

a) Porcentaje de células parabasales y células maduras: Se observó que el grupo de EHA de $500 \mathrm{mg} / \mathrm{kg}(80,37 \%)$ tuvo una reducción significativa en promedio de sus células parabasales en comparación con el grupo de las OVX tratadas con suero $(96,80 \%) p=0,006$.

b) Maduración celular Vs Aplicación de EHA: Al aplicar la prueba de Chi Cuadrado se encontró una asociación significativa entre dar tratamiento de EHA y maduración de las células vaginales; con un $p=0,012$; $\alpha=0,05$. Según lo obtenido pudimos evidenciar que las ratas OVX tratadas con suero no tuvieron maduración celular (Tabla 1).

\section{Estudio del tejido óseo}

a) Número de Trabéculas por campo en la metáfisis proximal de la tibia: Al comparar las medias de los grupos encontramos que las ratas ovariectomizadas tratadas con EHA de $10 \mathrm{mg} / \mathrm{kg}$ tuvieron un mayor número de trabéculas $(16,55 \pm 6,06)$ siendo superior a las ratas OVX tratadas con suero $(11,88 \pm 5,27)$, pero esta diferencia no es estadísticamente significativa.

b) Proporción de Trabéculas (\%) en la metáfisis proximal de la tibia: Este parámetro evalúa la superficie que ocupan las trabéculas en la zona metafisiaria de la tibia

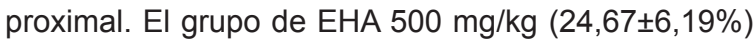

Tabla 1. Aplicación del EHA versus maduración celular en el frotis vaginal de ratas ovariectomizadas.

\begin{tabular}{|c|c|c|c|c|}
\hline & \multirow{2}{*}{ SI } & \multicolumn{2}{|c|}{$\begin{array}{l}\text { MADURACIÓN } \\
\text { CELULAR }\end{array}$} & \multirow[t]{2}{*}{ Total } \\
\hline & & NO & & \\
\hline \multirow{5}{*}{$\begin{array}{l}\text { APLICACIÓN } \\
\text { DE EHA }\end{array}$} & OVX + SUERO & $\begin{array}{c}0 \\
0 \%\end{array}$ & $\begin{array}{c}9 \\
39,1 \%\end{array}$ & $\begin{array}{c}9 \\
25 \%\end{array}$ \\
\hline & $O V X+E H A 10 \mathrm{mg} / \mathrm{kg}$ & $\begin{array}{c}2 \\
15,4 \%\end{array}$ & $\begin{array}{c}7 \\
30,4 \%\end{array}$ & $\begin{array}{c}9 \\
25 \%\end{array}$ \\
\hline & $O V X+E H A 100 \mathrm{mg} / \mathrm{kg}$ & $\begin{array}{c}5 \\
38,5 \%\end{array}$ & $\begin{array}{c}4 \\
17,4 \%\end{array}$ & $\begin{array}{c}9 \\
25 \%\end{array}$ \\
\hline & $O V X+E H A 500 \mathrm{mg} / \mathrm{kg}$ & $\begin{array}{c}6 \\
46,2 \%\end{array}$ & $\begin{array}{c}3 \\
13 \%\end{array}$ & $\begin{array}{c}9 \\
25 \%\end{array}$ \\
\hline & Total & $100 \%$ & $100 \%$ & $100 \%$ \\
\hline
\end{tabular}




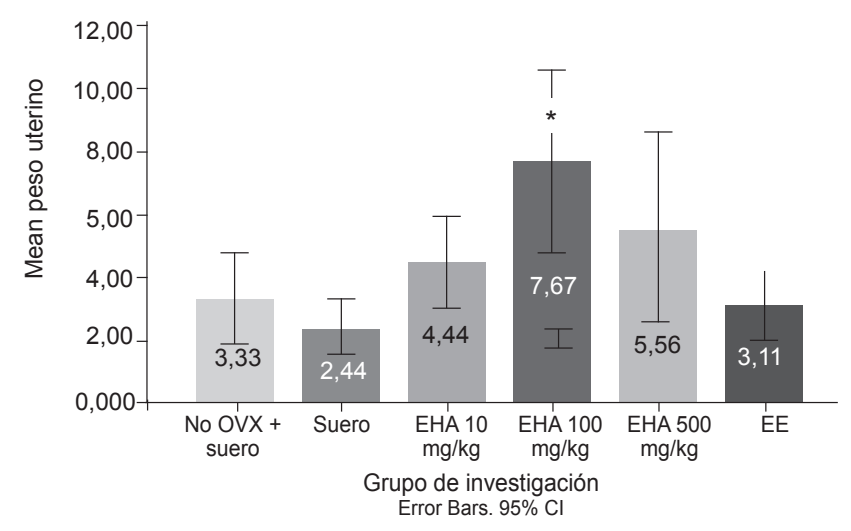

Figura 1. Número de osteoblastos $x$ campo $(40 \mathrm{X})$

$\left.{ }^{*}\right)$ Se observa diferencia estadísticamente significativa

fue superior a las ratas OVX tratadas con suero $(24,29 \pm 6,25 \%)$, esta diferencia no es estadísticamente significativa.

c) Grosor Trabecular $(\mu \mathrm{m})$ en la metáfisis proximal de la tibia en ratas ovariectomizadas: El grosor trabecular es uno de los parámetros afectados por la deficiencia de estrógenos; se encontró que las ratas tratadas con EHA $500 \mathrm{mg} / \mathrm{kg}(63,33 \pm 11,59 \mu \mathrm{m})$ fue superior a las OVX tratadas con suero fisiológico $(60,67 \pm 5,00 \mu \mathrm{m})$; no se encontró diferencia significativa.

d) Número de Osteoblastos, en las trabéculas de la metáfisis proximal de la tibia: El conteo de los osteoblastos de los grupos tratados con EHA fueron superior a las OVX tratadas con suero (media: 2,44 \pm 1,13), pero solo el grupo de EHA $100 \mathrm{mg} / \mathrm{kg}$ (media: 7,66 $\pm 3,80$ ) tuvo un incremento significativo de osteoblastos, según la prueba post hoc de Dunn (Gráfico 5).

\section{Peso uterino $(g)$}

El peso uterino del grupo de ratas $\sin \operatorname{OVX}(0,034 \pm 0,090)$ y el grupo de OVX tratadas con EE $(0,396 \pm 0,089)$ tuvieron un peso uterino 3 veces superiores a los grupos de OVX tratadas con suero $(0,095 \pm 0,022)$, EHA $10 \mathrm{mg} /$ $\mathrm{kg}(0,080 \pm 0,014)$, EHA $100 \mathrm{mg} / \mathrm{kg}(0,089 \pm 0,008)$ y EHA $500 \mathrm{mg} / \mathrm{kg}(0,109 \pm 0,018)$. Entre los grupos de EHA, el

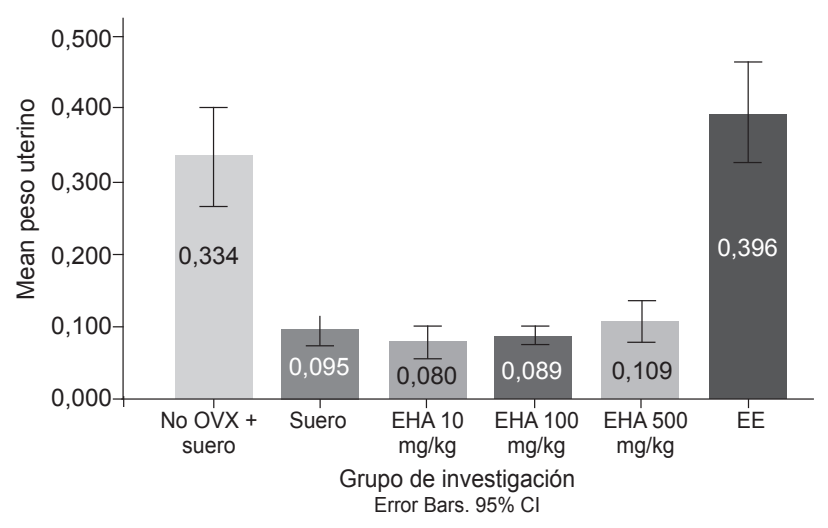

Figura 2. Peso del útero (gr) grupo de $500 \mathrm{mg} / \mathrm{kg}$ tuvo un valor ligeramente superior con respecto a las dosis inferiores (Gráfico 6).

\section{DISCUSIÓN}

El aguaje es un fruto selvático rico en nutrientes, dentro de los cuales destaca un fitoestrógeno llamado biochanina A, la cual produce un metabolito llamado genisteína la cual se ha demostrado que posee diversas propiedades que tendrían un gran impacto en la salud humana, como en la etapa postmenopáusica.

En el estudio del frotis vaginal se encontró una asociación significativa entre dar tratamiento de EHA y maduración de las células vaginales. En relación a los obtenido Cherdshewasart y col hallaron resultados positivos en las células vaginales de ratas ovariectomizadas al usar una planta llamada Pueraria Mirifica (rica en fitoestrógenos), cuya acción post-tratamiento en las células vaginales depende de la dosis usada ${ }^{11}$. De la misma forma Chiechi y col en un ensayo clínico con mujeres postmenopáusicas, llegaron a la conclusión de que una dieta rica en soja es eficaz en el aumento de los índices de maduración de las células vaginales ${ }^{12-13}$.

En el estudio del hueso trabecular no se encontraron diferencias significativas entre los grupos tratados con EHA y el grupo de Ovx tratadas con suero; sin embargo el grupo que recibió EHA de $500 \mathrm{mg} / \mathrm{kg}$ obtuvo mejores resultados en la proporción y grosor trabecular con respecto al grupo de Ovx que recibieron suero. A pesar de que los resultados no fueron estadísticamente significativos otros estudios realizados por Wang y col afirman que el uso de genisteína extraída de una planta medicinal china llamada Huaijiao Sophora japonica-Leguminosae en ratas ovariectomizadas obtuvo efectos favorables en el área y grosor trabecular del fémur ${ }^{14}$. En el número de osteoblastos se halló que los grupos que recibieron EHA tuvieron valores superiores al grupo de OVX tratadas con suero. Estos resultados se pueden deber, como se explica en un estudio de Lee y Choj, a que la Biochanina A influye significativamente en el crecimiento de las células osteoblásticas MC3T3-E1 y en la disminución de la producción de TNF-a en los osteoblastos, la cual está. relacionado a la actividad osteoclastogénica ${ }^{15-16}$.

El último parámetro de medición en el estudio fue el peso uterino encontrándose que los grupos OVX tratados con aguaje tuvieron un peso uterino muy similar a las OVX tratadas con suero, lo cual refleja que no hay una actividad estrogénica a este nivel; por el contrario el grupo de OVX que recibió etinilestradiol tuvo un peso uterino muy superior, observándose un efecto muy notorio en este órgano. En acorde Gallo y col. realizaron un estudio sobre el efecto del extracto estandarizado de soya en útero de ratas ovariectomizadas; encontrando en el peso uterino una reducción significativa además d atrofia en endometrio; en cambio el grupo de OVX que recibió $17 \beta$ estradiol tuvo un incremento significativo del peso uterino además de hiperplasia endometrial. ${ }^{17}$ También Schrepfer 
y col estudiaron el efecto de la Biochanina A en la cual no se evidenció crecimiento uterino ni cambios en su estudio histológico ${ }^{18}$.

Según nuestros resultados demostramos que el fruto de aguaje tiene actividad estrogénica en ratas ovariectomizadas; demostrándose a nivel de la células vaginales, a nivel óseo en los osteoblastos aunque no presentó actividad estrogénica significativa en el hueso trabecular. No se encontró actividad estrogénica en el peso uterino de ratas ovariectomizadas tratadas con ninguna de las dosis del Extracto hidroalcohólico del fruto de aguaje.

Es importante resaltar sus efectos positivos en la salud y como profesionales de la salud nos permite tener un fundamento científico para orientar e impulsar el consumo de este producto natural, en especial a la mujer postmenopáusica.

\section{AGRADECIMIENTOS}

A nuestras familias por su apoyo incondicional y su cariño; a todos los profesionales que nos apoyaron desde el inicio hasta la culminación de esta investigación.

\section{REFERENCIAS BIBLIOGRÁFICAS}

1. Paván $\mathrm{R}$, Mancini J, Del Castillo D, Alvarado L. Caracterización química y estabilidad oxidativa de los aceites de tres morfotipos de Mauritia flexuosa L. f., de la Amazonia Peruana. Laboratory of Natural Bioactive Substances PerúLaboratory of lipids Brazil. 2010; 61(4): 390-397.

2. Cusco C. Determinación de los compuestos fenólicos presentes en el extracto metanólico de la pulpa del fruto Mauritia Flexuosa L. "aguaje” procedente de Tarapoto-San Martin y su efecto sobre el nivel de estradiol en ratas hembras normales. [Tesis Magisterial] Perú. Universidad Nacional Mayor de San Marcos-Facultad de Farmacia y Bioquímica. 2009.

3. Benvenuto R, Garay J. Los fitoestrógenos ¿Alternativa a la THR en el climaterio?. Fronteras en Obstetricia y Ginecología 2002; 2(1): 49-60.

4. Haya J, Camil C, Perez T. Fitoestrógenos: conocimientos básicos y utilidad clínica. Hospital Universitario Santa Cristina (Madrid). Toko-Gin Pract 2002; 61(6):337-363.
5. Becerra F. Fitoestrógenos: clasificación y metabolismo. Editorial Diaz de Santos, Madrid 2003: 266-275.

6. Varangot J. Las pruebas de actividad de las substancias estrogénicas en la mujer: Aplicación al estudio del etinilestradiol. Rev Ginecol Obstet Mexicana 2007;75:172-177.

7. Zeni S, Gomez C, Di G. El Olpadronato previene la pérdida del hueso cortical y trabecular inducida por dosis suprafisiológicas de tiroxina en ratas ovarectomizadas. Medicina (Buenos Aires) 1998; 58: 453-457.

8. Arjmandi B. col.Dietary Soybean Protein Prevents Bone Loss in an Ovariectomized Rat Model of Osteoporosis. The Journal of Nutriction 2010: 161-167.

9. Close B, Banister K, Baumans V, Bernoth E, Bromage N, Bunyan J, Erhardt W, y col. Recomendaciones para la eutanasia de los animales de experimentación. Lab Animal 1997;31;1-32.

10. Cherdshewasart W, Kitsamia Y, Malavijitnond S. Evaluation of the estrogenic activity of the wild Pueraria mirifica by vaginal cornification assay. J Reprod Dev 2007; 53(2): 385-93.

11. Carbonel A, et al. The soybean concentrated extract proliferates the vagina of adult rats. Department of Morphology and Genetics, University of Sao Paulo, Brazil 2011; 18 (1) 93-101.

12. Chiechi L, Putignano G, Guerrea V, Shiavelli MP. The effect of a soy rich diet on the vaginal epithelium in postmenopause: a randomized double blind trial. J Reprod; 45(4):241-6.

13. Wang ZL, Sun JY, Wang DN, Xie YH, Wang SW, Zhao WM. Pharmacological studies of the large-scaled purified genistein fron Huaijiao (Sophora japonica-leguminosae) on antiosteoporosis.Phytomedicine 2006; 13(9-10): 718-23.

14. Lee K, Choj E. Biochanin A Stimulates osteoblastic differentiation and inhibits hidrogen peroxide-induced production of inflammatory mediators in MC3T3-E1 cells. Biol. Pharm. Bull Japan 2005; 28 (10): 1948-1953.

15. Karieb S, Fox SW. Phytoestrogens directly inhibit TNF- $\alpha$-induced bone resorption in RAW264.7 cells by suppressing c-fos-induced NFATc1 expression. Int J Mol Med. 2009;23(2):297-301.

16. Gallo D, Zannoni G, Apollonio P, Martinelli E, Morazzoni P, Bombardelli E, Scambia G. Characterization of the pharmacologic profile of a standardized soy extract in the ovariectomized rat model of menopause: effects on bone, uterus, and lipid profile. The Journal of The North American Menopause Society 2005; 12 (5): 589-600.

17. Schrepfer S, Deuse T, Schafer H, Koch F, Braendle W, Reichenspurner $\mathrm{H}$. The phytoestrogen Biochanin A weakens acute cardiac allograft rejection without affecting the reproductive system. Transplant Inmunology Alemania 2005; 15: 45 